\title{
Oral presentation of paraneoplastic pemphigus as the first sign of tonsillar HPV associated squamous cell carcinoma. A case report.
}

\author{
Jan Liska ${ }^{a}$, Veronika Liskova ${ }^{a}$, Ondrej Trckab , Ondrej Ondic, ${ }^{c, d}$ Lukas Hauera, Jan Baxae , Denisa Kacerovska, ${ }^{c, d}$
}

\begin{abstract}
Background. Paraneoplastic pemphigus (PNP) in the oral cavity is a rare variant of blistering pemphigus disease closely associated with mostly malignant tumors. The diagnosis may even precede an underlying malignancy enabling early detection. Here, we describe a previously unreported case of PNP associated with HPV-related tonsillar squamous cell carcinoma.

Methods and Results. A 50-year-old woman was referred to a dentist because of painful oral lesions resembling aphthae major and minor. Later, blisters appeared and an incisional biopsy was performed. Histological examination revealed an unusual coexistence of subepithelial and intraepithelial blisters raising suspicion of paraneoplastic pemphigus. The patient underwent 18F-FDG PET/MRI, showing a metabolically active process in the left palatal tonsil. Diagnostic biopsy revealed HPV type 16 associated tonsillar squamous cell carcinoma. A left tonsillectomy with elective left-sided neck dissection was performed. The postoperative period was complicated by bilateral fluidothorax. Two weeks after radical tumor removal, the mucosal and skin lesions of PNP disappeared. The patient currently shows no evidence of recurrence either of malignancy or PNP eight months after the surgery.

Conclusion. PNP is a rare autoimmune blistering disease characterized by polymorphous cutaneous and mucosal lesions associated with internal neoplasms including HPV associated squamous cell carcinoma of a tonsil. In order to identify an occult malignancy, a whole-body PET/CT or PET/MRI scan is recommended. Rarely, accurate patient management may depend on the dentist being familiar with this entity and on interdisciplinary cooperation involving dermatologist, radiologist, pathologist, and pneumologist. A strict patient follow-up is indicated.
\end{abstract}

Key words: paraneoplastic pemphigus, oral mucosa lesions; paraneoplastic autoimmune multiorgan syndrome, tonsillar carcinoma HPV associated, pulmonary complications, 18F-FDG PET/MRI

Received: April 16, 2021; Revised: June 9, 2021; Accepted: June 10, 2021; Available online: June 28, 2021

https://doi.org/10.5507/bp.2021.039

(c) 2022 The Authors; https://creativecommons.org/licenses/by/4.0/

${ }^{a}$ Department of Dentistry, University Hospital and Faculty of Medicine in Pilsen, Charles University, Alej Svobody 80, 30460 Pilsen, Czech Republic

${ }^{b}$ Department of Otorhinolaryngology, University Hospital and Faculty of Medicine in Pilsen, Charles University, Alej Svobody 80,30460 Pilsen, Czech Republic

'Sikl's Department of Pathology, Faculty of Medicine in Pilsen, Charles University, Ed. Benese 13, 30599 Pilsen, Czech Republic

${ }^{d}$ Biopticka Laboratory, Mikulasske namesti 628, 32600 Pilsen, Czech Republic

eDepartment of Imaging Methods, University Hospital and Faculty of Medicine in Pilsen, Charles University, Alej Svobody 80, 30460 Pilsen,

Czech Republic

Corresponding author: Lukas Hauer, e-mail: hauerl@fnplzen.cz

\section{INTRODUCTION}

Paraneoplastic pemphigus (PNP) in the oral cavity is a rare variant of blistering pemphigus disease closely associated with an underlying mostly malignant process. The delayed diagnosis usually complicates the management of PNP due to its variable clinical features. This paper describes an unusual case of PNP associated with HPV associated tonsillar squamous cell carcinoma. To the best of our knowledge, this is the first reported case in the English medical literature describing PNP associated with a carcinoma of the tonsils.

\section{CASE REPORT}

A 50-year-old woman with a medical history of recurrent aphthous stomatitis and herpes simplex labialis was referred to a dentist because of painful oral lesions. The symptoms started abruptly a few days previously, but she had felt unwell for several months. The patient presented with painful lesions on the right buccal mucosa reminiscent of one major aphtha and two minor aphthae (Fig. 1A). This was treated as a recurrent aphthous stomatitis using Er:Cr laser with mucosal 1000 micron tip and topical Dexamethasone solution, rinsing with Chlorhexidine $0.12 \%$ and Dexpanthenol solution. The lesional response for this treatment seemed optimal, but three weeks later, new lesions appeared on the left tongue margin, on the left buccal mucosa and the left half of the soft palate. This time, the lesion's features differed from 

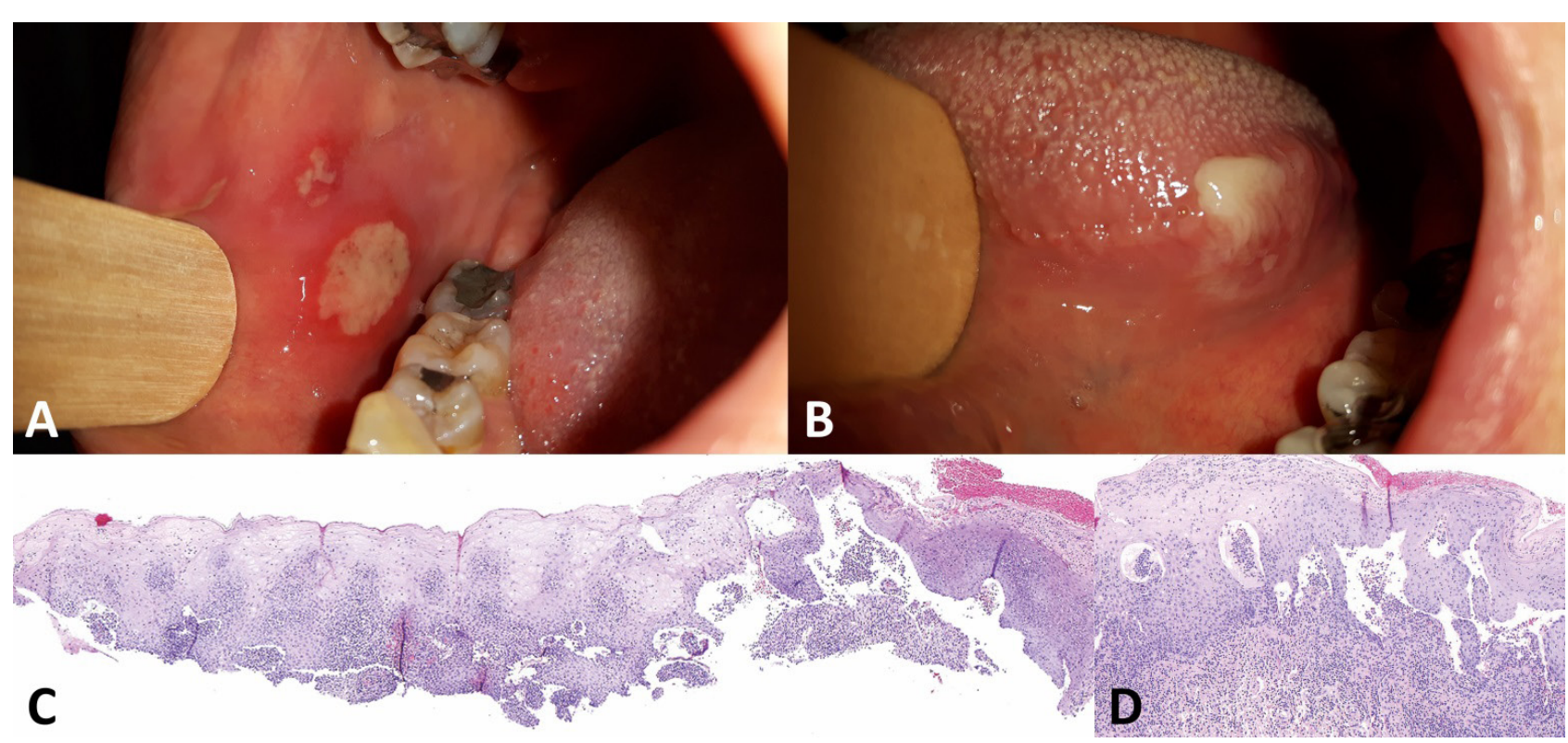

Fig. 1. (A) Primary lesion of the right buccal mucosa evaluated as a recurrence of aphthous stomatitis treated with local corticosteroid and laser therapy. (B) Sloughing blister at the left tongue margin. The same lesions appeared on the left buccal mucosa and labial mucosa of the lower lip. (C) Whole-mount of the lesion: hyperplastic epithelium with subepidermal clefting/ blisters and dense inflammatory infiltrate. (D) Close-up of the area corresponding to the finding of coexisting intraepidermal vesicles and subepidermal clefting with both neutrophils and eosinophils.

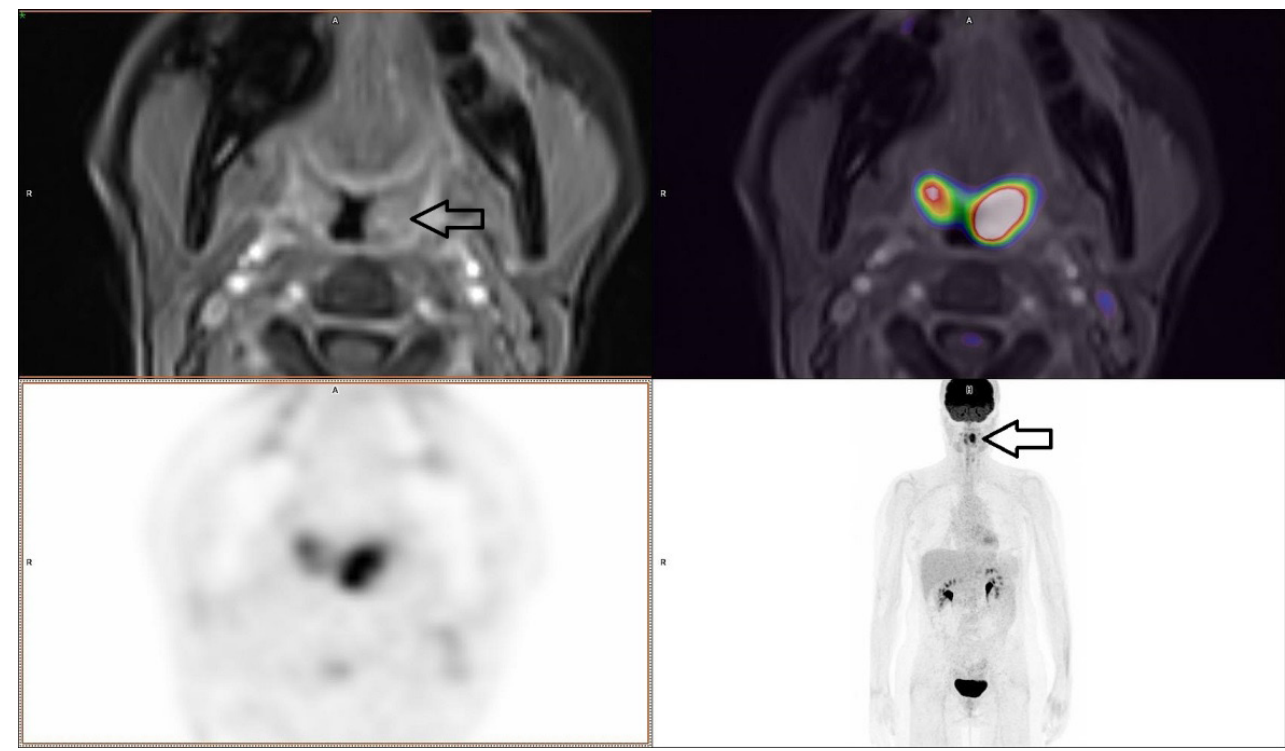

Fig. 2. 18F-FDG PET/MRI scan shows FDG signal accumulation at the left tonsil area subsequently histologically verified as left tonsillar carcinoma (arrow) - MRI in transversal view (left upper), PET image in transversal view (left lower), fused MRI and PET (right upper) and whole-body PET (right lower).

the previous one with no aphthous halo and characterized by sloughing blisters (Fig. 1B). Diagnostic excision from the buccal mucosa was performed. Histologically, subepithelial and intraepithelial blisters were identified, filled with mixed inflammatory cells dominated by neutrophils and eosinophils. Inconspicuous vacuolar alteration of the dermo-epidermal junction and exocytosis of small well-differentiated lymphocytes were also seen. The changes were considered consistent with PNP (Fig. 1C, 1D) although, direct immunofluorescence examination was negative. A few days later, skin lesions in the anogenital area followed the course. Also, new erosions had developed on the labial mucosa of the lower lip.

Due to histologic suspicion and progressing clinical symptoms, whole-body screening by $18 \mathrm{~F}-\mathrm{FDG}$ PET/MRI was performed. Enlarged left palatal tonsil with significantly increased FDG uptake was identified as a solitary lesion suspicious for malignancy (Fig. 2). Incisional biopsy from the tonsil confirmed the diagnosis of invasive moderately differentiated squamous cell carcinoma. 
Table 1. Paraneoplastic pemphigus diagnostic criteria modified by Camisa and Helm².

\begin{tabular}{ll}
\hline Signs & Features \\
\hline Major & Polymorphic mucocutaneous eruption \\
& Concurrent internal neoplasia \\
& $\begin{array}{l}\text { Serum antibodies with a specific } \\
\text { immunoprecipitation pattern }\end{array}$ \\
Minor & Histological evidence of acantholysis \\
& DIF showing intercellular and basement \\
& membrane staining \\
& IIF staining with rat bladder epithelium \\
\hline
\end{tabular}

DIF, direct immunofluorescence; IIF, indirect immunofluorescence

Additionally, molecular-genetic study revealed the presence of high-risk human papillomavirus type 16. A left tonsillectomy with elective left-sided neck dissection of levels II-IV was performed. No lymph node metastases were identified. Systemic therapy consisted of a prophylactic combination of two antibiotics, namely Amoxicillin and Metronidazole. After tonsillectomy, the mucosal and skin lesions healed within two weeks. There was no need for radiotherapy or chemotherapy due to the clear margin excision of the tumor. The postoperative period was complicated by bilateral fluidothorax $(650 \mathrm{~mL}$ on the right, $100 \mathrm{~mL}$ on the left side). The patient felt epigastric pain without breathing difficulties. Within three days, the pulmonary fluid was spontaneously reduced. The residual right-sided fluidothorax of $250 \mathrm{~mL}$ was evacuated. Cytologically, it contained eosinophilic leukocytes, reactive mesothelial cells, isolated lymphocytes and neutrophilic leukocytes. No malignant cells were present. At eight months clinico-radiological follow-up using 18F-FDG PET/MRI, there were no signs of recurrence both of malignancy nor PNP.

\section{DISCUSSION}

PNP is a rare variety of pemphigus blistering diseases described for the first time by Anhalt in 1990 (ref. $^{1}$ ), with its defining criteria revised by Camisa and Helms in 1993 (Table 1) (ref. $\left.{ }^{2,3}\right)$. This definition is based on the original description and criteria of paraneoplastic skin syndrome by $\mathrm{Curth}^{4}$. PNP is associated with benign and malignant tumors - mostly haemato-oncologic malignancies ${ }^{5-7}$, less frequently with carcinomas ${ }^{8}$, thymomas ${ }^{9}$ and mesenchymal tumors ${ }^{10,11}$.

This case report extends the spectrum of solid tumors associated with PNP adding HPV associated squamous cell carcinoma of a tonsil. In approximately one-third of reported cases, PNP preceded the diagnosis of malignancy ${ }^{12}$. Timely diagnosis of PNP may be hampered by polymorphous clinical and histological features of the lesions affecting the skin and oral mucosa including potentially malignant disorders per $s e^{13-15}$. Currently, at least seven clinical variants of PNP are recognized (i.e. ery- thema multiforme-like, pemphigoid-like, pemphigus-like, graft-versus-host disease-like, lichen planus pemphigoides / (erosive) lichen planus-like, cicatricial pemphigoid-like, linear IgA dermatosis-like) (ref. ${ }^{16}$ ). Oral involvement clinically presents as panstomatitis, resembling pemphigus vegetans, mucosal pemphigoid or/and erythema multiforme. In the oral cavity, multiple areas of erythema, irregular ulcerations or erosions ${ }^{17}$, sloughing blisters, or even vermilion crusts can be seen. Lips may show hemorrhagic crusts similar to erythema multiforme. The lesions are often very painful. The symptoms usually begin suddenly and are resistant to standard therapy. In a small number of patients, oropharyngeal lesions may develop without cutaneous involvement. In severe PNP cases, vaginal mucosa and mucosa of the respiratory tract can be affected.

In 2001, the paraneoplastic autoimmune multiorgan syndrome was introduced ${ }^{18}$. It is defined by the presence of circulating antibodies targeting different organs, namely lung, kidney, smooth and striated muscle, intestinal epithelium and thyroid gland. Antibodies target specifically desmoglein 3, periplakin and epiplakin ${ }^{19,20}$. A PNP usually confers an unfavourable prognosis. Some cases can be complicated by pulmonary disease, particularly bronchiolitis obliterans that may lead to respiratory failure, as described for the first time in 1999 (ref. ${ }^{21}$ ). Other features of PNP include eye lesions such as severe pseudomembranous conjunctivitis or/and corneal melting. The anogenital region can also be affected, as in the above-described case. The clinical course of PNP is challenging to manage. It is frequently complicated by infections, especially pneumonia and obliterating bronchiolitis. Most PNP cases are resistant to treatment. Thus, PNP mortality is high (up to $90 \%$ in untreated patients) and quite often not directly connected with underlying associated malignancy ${ }^{19,22}$. Early diagnosis of PNP and underlying tumor is essential for successful treatment. The whole-body FDG-PET/CT $\operatorname{scan}^{10}$ or FDG-PET/MRI, reported for the first time in the present paper, is recommended as an important part of diagnostic procedure and post-therapeutic follow-up. Systemic therapy consists of a variety of drugs and their combinations $^{23}$.

\section{CONCLUSION}

PNP is a rare autoimmune blistering disease characterized by polymorphous cutaneous and mucosal lesions associated with internal neoplasms including HPV associated squamous cell carcinoma of a tonsil. In order to identify an occult malignancy, a whole-body PET/CT or $\mathrm{PET} / \mathrm{MRI}$ scan is recommended. Rarely, accurate patient management may depend on the dentist being familiar with this entity and on interdisciplinary cooperation involving dermatologist, radiologist, pathologist, and pneumologist. A strict patient follow-up is indicated.

Author contributions: JL: manuscript writing, literature search, clinical data collection; VL, LH: clinical data collection, literature search, review of the manuscript; OT: evaluation and revision of ENT issue; JB: PET/MRI acqui- 
sition and evaluation; OO: literature search, review of the manuscript, final approval; DK: histological assessment, review of the manuscript, final approval. All authors read and approved the final manuscript as submitted.

Conflict of Interest Statement: None declared.

\section{REFERENCES}

1. Anhalt GJ, Kim SC, Stanley JR, Korman NJ, Jabs DA, Kory M, Izumi H Ratrie H, Mutasim D, Ariss-Abdo L, Labib RS. Paraneoplastic pemphigus. An autoimmune mucocutaneous disease associated with neoplasia. N Engl J Med 1990;323(25):1729-35.

2. Camisa C, Helm TN. Paraneoplastic pemphigus is a distinct neoplasia-induced autoimmune disease. Arch Dermatol 1993;129(7):883-6.

3. Helm TN, Camisa C, Valenzuela R, Allen CM. Paraneoplastic pemphigus: A distinct autoimmune vesiculobullous disorder associated with neoplasia. Oral Surg Oral Med Oral Pathol 1993;75(2):209-13.

4. Curth HO. Cancer associated with acanthosis nigricans: review of literature and report of a case of acanthosis nigricans with cancer of the breast. Arch Surg 1943;47(6):517-52.

5. Maruta CW, Miyamoto D, Aoki V, de Carvalho RGR, Cunha BM, Sant CG. Paraneoplastic pemphigus: A clinical, laboratorial and therapeutic overview. An Bras Dermatol 2019;94(4):388-98.

6. Cao L, Wang F, Du XY, Zhu HY, Wang L, Xu W, Li JY, Fan L. Chronic lymphocytic leukemia-associated paraneoplastic pemphigus: potential cause and therapeutic strategies. Sci Rep 2020;10(1):16357.

7. Ouedraogo E, Gottlieb J, de Masson A, Lepelletier C, Jachiet M, Salle de Chou C, Galicier L, Bagot M, Chevret S, Bouaziz JD. Risk factors for death and survival in paraneoplastic pemphigus associated with hematologic malignancies in adults. J Am Acad Dermato 2019:80(6):1544-49.

8. Kaplan I, Hodak E, Ackerman L, Mimouni D, Anhalt GJ, Calderon S. Neoplasms associated with paraneoplastic pemphigus: a review with emphasis on non-hematologic malignancy and oral mucosal manifestations. Oral Oncol 2004;40(6):553-62.

9. Khurana R, Sharma S, Kumar S, Deshpande AA, Wadhwa D, Agasty S. Paraneoplastic pemphigus associated with a pericardial ectopic thymoma. J Card Surg 2020;35(11):3141-44.
10. Chen X, Fu Z, Yang X, Li Q. 18F-FDG PET/CT in follicular dendritic cell sarcoma with paraneoplastic pemphigus as the first manifestation. Clin Nucl Med 2020;45(7):572-74.

11. Ghandi N, Ghanadan A, Azizian MR, Hejazi P, Aghazadeh N, Tavousi $\mathrm{P}$, Daneshpazhooh M. Paraneoplastic pemphigus associated with inflammatory myofibroblastic tumour of the mediastinum: A favourable response to treatment and review of the literature. Australas J Dermatol 2015;56(2):120-3.

12. Neville BW, Damm DD, Allen CM, Bouquot JE, editors. Oral and maxillofacial pathology. 3rd ed. St. Louis: Saunders; 2009

13. Bolognia JL, Schaffer JV, Cerroni L, editors. Dermatology. 4th ed. Philadelphia: Elsevier; 2018.

14. Callen JP, Jorizzo JL, Zone JJ, Piette WW, Rosenbach MA, Vleugels RA. Dermatological signs of systemic diseases. 5th ed. Toronto: Elsevier; 2017.

15. Warnakulasuriya S. Clinical features and presentation of oral potentially malignant disorders. Oral Surg Oral Med Oral Pathol Oral Radiol 2018;125(6):582-90.

16. Patterson JW. Weedon's skin pathology. 5th ed. Amsterdam: Elsevier; 2020.

17. Kartan S, Shi VY, Clark AK, Chan LS. Paraneoplastic Pemphigus and Autoimmune Blistering Diseases Associated with Neoplasm: Characteristics, Diagnosis, Associated Neoplasms, Proposed Pathogenesis, Treatment. Am J Clin Dermatol 2017;18(1):105-26.

18. Amber KT, Valdebran M, Grando SA. Paraneoplastic autoimmune multiorgan syndrome (PAMS): Beyond the single phenotype of paraneoplastic pemphigus. Autoimmun Rev 2018;17(10):1002-10.

19. Yong AA, Tey HL. Paraneoplastic pemphigus. Australas J Dermatol 2013;54(4):241-50.

20. Carey B, Setterfield J. Mucous membrane pemphigoid and oral blistering diseases. Clin Exp Dermatol 2019;44(7):732-39.

21. Nousari HC, Deterding R, Wojtczack H, Aho S, Uitto J, Hashimoto T, Anhalt GJ. The mechanism of respiratory failure in paraneoplastic pemphigus. N Engl J Med 1999;340(18):1406-10.

22. Paolino G, Didona D, Magliulo G, lannella G, Didona B, Mercuri SR, Moliterni E, Donati M, Ciofalo A, Granata G, Ranuzzi P, Falasca V, Calvieri S. Paraneoplastic Pemphigus: Insight into the Autoimmune Pathogenesis, Clinical Features and Therapy. Int J Mol Sci 2017;18(12):2532

23. Tirado-Sánchez A, Bonifaz A. Paraneoplastic Pemphigus. A LifeThreatening Autoimmune Blistering Disease. Actas Dermosifiliogr 2017; 108(10):902-10. 